\title{
Ocupación y desastres: la formación de terapeutas ocupacionales para la prevención y la reducción del riesgo
}

\section{Occupation and disasters: Occupational therapists' training for prevention and risk reduction}

\author{
Rosana Verónica Ariño ${ }^{1}$ \\ Mariana Margarita Boffelli² \\ Carla Rosana Boggio ${ }^{3}$ \\ Daniela Chiapessoni ${ }^{4}$ \\ Mauro Alejandro Demichelis ${ }^{5}$ \\ María de los Milagros Dermiyi ${ }^{6}$ \\ María del Carmen Heit ${ }^{7}$ \\ Cristian Alberto Quinteros ${ }^{8}$
}

Recibido: 20 agosto 2014 • Enviado para modificación: 29 noviembre 2014 • Aceptado: 25 junio 2015

Ariño, R.V., Boffelli, M.M., Boggio, C.R., Chiapessoni, D., Demichelis, M.A., Dermiyi, M.M., Heit, M.C. \& Quinteros, C.A. (2015). Ocupación y desastres: la formación de terapeutas ocupacionales para la prevención y la reducción del riesgo. Revista Ocupación Humana, 15(1), pp. 19 - 34.

Resumen: Los desastres son un tema de relevancia y actualidad a nivel mundial y latinoamericano. Este artículo presenta el análisis de la temática ocupacional, realizada por los participantes del curso de posgrado: Intervención de Terapia Ocupacional en Emergencias y Desastres, impulsado por el equipo de investigación Intervención de Terapia Ocupacional en Catástrofes, de la Universidad Nacional del Litoral en Santa Fe, Argentina. Durante el curso se realizó una investigación educativa-empírica. La categoría ocupación se analizó desde los cambios en roles, rutinas y contextos en situaciones de desastres, y a partir de las experiencias de los participantes. Se identificó un nuevo escenario de intervención profesional y se planteó un ineludible posicionamiento político respecto a la ocupación como derecho, la justicia

\footnotetext{
${ }^{1}$ Licenciada en Terapia Ocupacional. Docente-investigadora, Universidad Nacional del Litoral, Santa Fe, Argentina.rosana.arino@gmail.com

2 Terapeuta Ocupacional. Docente-investigadora, Universidad Nacional del Litoral, Santa Fe, Argentina. boffelli@fbcb.unl.edu.ar

${ }^{3}$ Terapeuta Ocupacional. Docente-investigadora, Universidad Nacional del Litoral, Santa Fe, Argentina. crboggio@yahoo.com.ar

${ }^{4}$ Terapeuta Ocupacional. Docente-investigadora, Universidad Nacional del Litoral, Santa Fe, Argentina. danielachiapessoni@hotmail.com

${ }^{5}$ Licenciado en Terapia Ocupacional. Magíster en Docencia Universitaria. Docente-investigador, Universidad Nacional del Litoral, Santa Fe, Argentina.m_demichelis@hotmail.com

${ }^{6}$ Terapeuta Ocupacional. Especialista en Docencia Universitaria. Especialista en Docencia en Entornos Virtuales. Docente-investigadora, Universidad Nacional del Litoral y Universidad Nacional de Quilmes, Santa Fe y Buenos Aires, respectivamente, Argentina.milagros.demiryi@gmail.com

${ }^{7}$ Licenciada en Terapia Ocupacional. Investigadora, Universidad Nacional del Litoral, Santa Fe, Argentina. mariquiten@hotmail.com

${ }^{8}$ Analista en Informática Aplicada. Especialista en Informática Educativa. Docente-investigador, Universidad Nacional del Litoral, Santa Fe - Argentina. cquinteros@unl.edu.ar
} 
ocupacional, las diferentes formas de injusticia ocupacional, como el apartheid ocupacional, que devienen y derivan de situaciones de desastres. Captar las características centrales de las ocupaciones, en las diferentes instancias del ciclo de desastre, posibilitó proponer servicios y acciones para cada situación. Se elaboraron propuestas de intervención con énfasis en lo local. Se aportó a consolidar una identidad profesional centrada en el valor y la potencialidad de las ocupaciones, y en la reivindicación de las mismas como una necesidad y un derecho humano.

Palabras clave: Capacitación profesional, Terapia Ocupacional, ocupación humana, desastres.

Abstract: Disasters are a current and relevant issue in Latin America and worldwide. This article introduces an occupational subject analysis, made by the participants of the international postgraduate online-course: Occupational Therapy Intervention in Disasters and Emergencies, initiated by the research team of 'Occupational Therapy Intervention in Disasters' of the Universidad Nacional del Litoral in Santa Fe, Argentina. An empirical investigation was made throughout the course. The category of 'occupation' was analyzed by considering the changes that emerged from disasters such as changes in roles, routines, and contexts, and the participants' experiences. The participants identified a new scenario of professional intervention, and its unavoidable political position, when considering occupation as a right, occupational justice, and the many forms of occupational injustice such as occupational apartheid, that occur and drift from disaster situations. Proposing services and actions for each situation was possible through an understanding of the main characteristics of occupations in different stages of the cycle of the disaster. Proposals for intervention emphasized a local approach. This article contributes to strengthen the professional identity focused on the value and potentiality of occupations, and the recognition of them as a necessity and a human right.

Keywords: Professional training, Occupational Therapy, human occupation, disasters.

\section{Introducción}

Las recurrentes situaciones de desastres vividas en el mundo en general, y particularmente en América Latina y El Caribe, ponen de manifiesto la urgente necesidad de que las comunidades y los profesionales vinculados al ámbito socio-sanitario se preparen para el trabajo en los diferentes momentos del ciclo de los desastres.

El curso Intervención de Terapia Ocupacional en Emergencias y Desastres, surge como respuesta a los vacíos existentes en la formación profesional de Terapia Ocupacional. Tales vacíos generan parcialidades en el razonamiento, inicio y sostenimiento de interven- ciones desde el campo disciplinar en el área, y se inscriben en un contexto global en donde crece la importancia de dicho conocimiento, y a la vez, se desarrollan experiencias educativas que utilizan las tecnologías de la información y la comunicación.

El curso se organizó para los profesionales de Terapia Ocupacional de América Latina y El Caribe, dados los acontecimientos que a diario ocurren en esta amplia región por la vulnerabilidad geo-social en la que se encuentra inmersa, por el incremento de eventos catastróficos, por su frecuencia de aparición y por la perspectiva de continuidad a futuro. Lo anterior sumado 
a las demandas recibidas por el equipo de parte de colegas de distintos países que conforman nuestro continente, y a partir de la identificación de que los mismos no están lo suficientemente preparados para afrontar y dar respuesta a situaciones de desastres.

Existen diversas formas de definir el riesgo, se trata de un término polisémico; aun así, la idea de riesgo asociada a la probabilidad de que una amenaza se convierta en desastre, es compartida por todas las perspectivas; de igual modo, la incertidumbre e inseguridad de la ocurrencia y de sus consecuencias.

El riesgo de desastres es una construcción social que implica un proceso acumulativo en el que se combinan múltiples factores que intervienen en el desarrollo y la construcción de nuestro hábitat (Valdés, 2006).

Desde la perspectiva de la teoría social (Giddens, 1993), el riesgo es una categoría asociada a la modernidad y sus consecuencias: la caracterización de sociedades del riesgo expresada en el aumento de estados débiles y frágiles; la creciente afectación a los derechos humanos y los precarios avances en la reducción de la pobreza y en el mejoramiento de los niveles de bienestar; el enfoque político-ecológico que enfatiza en las desigualdades producidas en la distribución espacial y social de riesgos y vulnerabilidades. En este sentido Blaikie, Cannon, David \& Wisner (1996) y Lavell (2005), desde la escuela de vulnerabilidad, y Beck (2000, 2002), desde la teoría social del riesgo, han desarrollado aportes para la comprensión de estos fenómenos.
Ninguna situación de desastre o catástrofe puede ser entendida de manera aislada o como fenómeno exclusivamente natural, sino en el contexto de las profundas alteraciones producidas por el hombre en el ambiente y en relación a las políticas implementadas. La vulnerabilidad social, entendida como la potencialidad de personas, grupos, comunidades y sistemas que pueden ser afectados, dañados o perjudicados, es una de las dimensiones del riesgo que caracteriza particularmente a los países pobres y a los más pobres de cada país, a la vez que afecta la gestión del riesgo como anticipación del futuro.

Ante estos escenarios, uno de los aportes más importantes es la preparación de las poblaciones y comunidades para afrontar o anticiparse a tales eventos, "no estar preparados para lo inesperado es disculpar a los responsables" (Gascón, 2005, p.12). Hay países que están tomando medidas para prepararse ante las posibles adversidades que acarrearían; los tiempos de preparación suelen ser los posteriores a la ocurrencia de estos fenómenos.

Lo hasta aquí dicho, constituye una breve presentación de los fundamentos de la propuesta del curso, enmarcado en un proyecto de investigación.

\section{Metodología}

El proyecto de investigación: Diseño, desarrollo y evaluación de un curso de capacitación virtual sobre intervención en catástrofes, fue desarrollado por el equipo de investigación Intervención de Terapia Ocupacional en Catástrofes (INTOCA), de la Universidad Nacional del Litoral (UNL) en Santa Fe, Argen- 
tina. La investigación se realiza en el marco de los Cursos de acción, investigación y desarrollo $(\mathrm{CAI}+\mathrm{D})$, política que propone la UNL para promover las actividades científico tecnológicas de jóvenes investigadores y grupos de trabajo, favoreciendo además la asociación de grupos y unidades académicas y la articulación, tanto de líneas de investigación, como de disciplinas y campos del saber.

Este proyecto fue de tipo educativoempírico, con énfasis en la evaluación como ejercicio del empoderamiento de los propios participantes desde los principios del paradigma participativo (Fetterman, 2001). Esta elección metodológica radica en la compatibilidad con el propósito del estudio, la captación del punto de vista de los participantes de la experiencia, el contexto natural en que se desarrolló y el tratamiento de los datos.

El curso, desarrollado en el marco de la investigación antes mencionada, fue de cuarto nivel (curso de posgrado), con una carga horaria de 160 horas. El diseño comprendió cinco módulos mensuales de 32 horas cada uno, con actividades semanales de 8 horas. Fue acreditado por la Universidad Nacional del Litoral mediante la aprobación del Consejo Directivo de la Facultad de Bioquímica y Ciencias Biológicas, bajo Resolución C.D. № 852.

Los módulos fueron estructurados de la siguiente manera:

Módulo I: Introducción a la problemática de desastres y emergencias.

Módulo II: Gestión integral de riesgo.

Módulo III: Escenarios de riesgos.
Módulo IV: Manejo de crisis.

Módulo V: Recuperación y reconstrucción.

La etapa del diseño del curso comprendió las tareas de:

- Organización general y soporte logístico.

- Diseño curricular: estructura y calendarización.

- Diseño didáctico: objetivos, secuencia de contenidos, estrategias y evaluación de los aprendizajes.

- Producción de contenidos: materiales, elementos textuales y gráficos, enlaces.

- Aspectos y condiciones tecnológicas: requisitos, interfaz, accesibilidad.

- Mediación pedagógica: comunicación e interacciones.

Cada módulo contó con una evaluación de los aprendizajes (obligatoria) y actividades optativas.

En el desarrollo del curso participaron: el equipo de investigación, los equipos de especialistas y técnicos del Centro Multimedial de Educación a Distancia (CEMED) (docentes, pedagogos, comunicadores, diseñadores, programadores y personal técnico-administrativo). Para el dictado de los módulos se gestionó la participación de docentes invitados argentinos y extranjeros.

Se trató de la primera iniciativa sobre el tema en la profesión a nivel nacional e internacional. La propuesta de formación de recursos resultó innova- 
dora, tanto por la vigencia de la temática, como por la modalidad virtual. Por haberse desarrollado en el marco de un proyecto de investigación, posee la potencialidad de producir y validar conocimientos científicos en un tema en el que se evidencian pocos adelantos y escasa producción.

El grupo inicial de participantes estuvo conformado por 17 terapeutas ocupacionales. Las nacionalidades y ubicaciones geográficas muestran el alcance latinoamericano de la propuesta; los participantes de Argentina a su vez, tenían diversas procedencias (Tabla 1).

El desempeño académico de los participantes se expresó en una calificación final promedio de 8,35 en escala de 10. La participación en foros de intercambio y actividades colaborativas fue de 4,5 intervenciones promedio (por cada participante y por módulo). Se registró una tendencia decreciente en los dos últimos módulos (M1:45 -
M5:30), según los alumnos por dedicar más tiempo a la lectura de materiales y actividades. La mayor participación y actitud pro-activa se produjo en las instancias de foros y actividades grupales; esto evidencia su efectividad para propiciar aprendizajes comprensivos (Huerta, 1976) y colaborativos, mediados por el entorno virtual. La calidad de las intervenciones y de los trabajos elaborados por los participantes constituyen evidencias de la adquisición de nuevos conocimientos y habilidades y son, a la vez, aportes para el desarrollo de esta área profesional emergente.

A continuación se presenta uno de los temas de debate y reflexión propuestos como actividad, y el análisis de los contenidos de las intervenciones y producciones de los participantes. El tema seleccionado para la presentación en este artículo es el de Ocupación y Desastres.

Tabla 1. Procedencia de los participantes

\begin{tabular}{|c|c|c|}
\hline País & Ciudad/Provincia & $\begin{array}{c}\text { № de } \\
\text { participantes }\end{array}$ \\
\hline \multicolumn{2}{|l|}{ Brasil } & 2 \\
\hline \multicolumn{2}{|c|}{ Colombia } & 1 \\
\hline \multicolumn{2}{|l|}{ Chile } & 2 \\
\hline \multicolumn{2}{|l|}{ Perú } & 1 \\
\hline \multicolumn{2}{|c|}{ México } & 1 \\
\hline \multirow{5}{*}{  } & Santa Fe, capital y provincia & 3 \\
\hline & Buenos Aires, capital y provincia & 4 \\
\hline & Córdoba & 1 \\
\hline & Mar del Plata & 1 \\
\hline & Santa Cruz & 1 \\
\hline \multicolumn{2}{|c|}{ Total Argentina } & 10 \\
\hline
\end{tabular}

Fuente. Elaboración propia. 


\section{Resultados y discusión}

\section{Cambios en las ocupaciones, roles y contextos ocupacionales}

La categoría ocupación atraviesa longitudinalmente y de manera variable el desarrollo completo del curso:

- En forma directa a partir de los relatos y las participaciones en los foros.

- Como categoría a analizar en la resolución de los trabajos finales de los módulos.

- En la forma de la propia participación de los alumnos en el curso.

En los siguientes extractos, tomados de los foros, se refleja en forma más o menos explícita la centralidad de la ocupación en sus diversas áreas:

"Me han llamado la atención la cantidad de damnificados desplazados de su hábitat y ocupación natural, tanto como la cantidad de años que Colombia se ve afectada por esta situación [desplazamiento forzado]...; como relata el colega, esta experiencia de Colombia me recuerda muchos de los conceptos tratados en el libro T.O Sin Fronteras, como es la injusticia ocupacional, al tener que dejar las actividades cotidianas y volver a reconstruir la historia en otras zonas obligado por otro, o el Apartheid Ocupacional, al no tener derecho al uso y goce de la ocupación..." (Colega de Chile, sobre relato de desplazamiento forzado en Colombia).

"...El hecho que esta villa por muchos años ha estado bajo el agua, impidiendo que las personas pu- dieran continuar con sus vidas en el lugar en el que habían construido su historia. Además del quiebre propio de la inundación, se produce la imposibilidad de volver a la normal cotidianeidad en los espacios comunes con otros..." (Inundación en Villa Epecuén-Cahue, provincia de Buenos Aires, Argentina, 1985).

“....Los afectados, al perder su fuente laboral... pierden parte de la vida.... Frente a la inundación de Villa Epecuén, los hoteleros y las personas que han desarrollado su fuente laboral en torno al turismo, sufren además un cambio en su estatus económico, dado que pierden sus bienes inmuebles y todas las inversiones que hicieron por años, lo que no es compensado con la indemnización que ofrece el gobierno..." (Inundación en Villa Epecuén-Cahue, provincia de Buenos Aires, Argentina, 1985).

Se reconoce recurrentemente el quiebre que los desastres producen en la vida de las personas y las poblaciones, no sólo en el período inmediato a la ocurrencia del evento, sino a mediano y largo plazo. Los participantes en sus reflexiones abordan este aspecto enumerando algunos de los modos en que las ocupaciones, su forma, su periodicidad, su poder central como organizadoras de la cotidianeidad, se desploman ante el nuevo orden de cosas. También se reconoce que los desastres impactan en las prioridades otorgadas a las ocupaciones. Cada matiz abordado en el análisis que sigue deriva inmediatamente a la reflexión acerca de 
qué nuevas formas, funciones y significados pueden tomar las ocupaciones, incluso las más habituales, cuando el entorno ha sufrido modificaciones tan sustanciales. En su análisis de la Gran Nevada de Santa Cruz de 1995, ${ }^{9}$ una participante sistematiza las consecuencias; muchas de ellas, refieren a cambios ocupacionales:

“...Cambios en la vida cotidiana: restricción para circular en calles y rutas, cese de actividades económicas, sociales y culturales, educativas, recreativas. Pedido de salir de los hogares solo en casos imprescindibles. Dificultades para el ingreso de alimentos por vía terrestre. Cese de transportes aéreos. Cortes de luz. Falta de calefacción adecuada. El sistema de salud reducido a las emergencias...rumores en relación a la escasez de alimentos que disparan conductas individualistas al extremo, en convivencia con respuestas colectivas de cooperación y creatividad. Temor al aislamiento prolongado, vivencias de encierro, necesidad de poner a resguardo a hijos o familias, intentando por todos los medios salir de la provincia, angustia existencial (ipor qué me vine tan lejos... vale la pena...?), acopio excesivo de alimentos, nafta, etc..." (Gran Nevada en Santa Cruz, provincia de Argentina, 1995).

Los cambios copernicanos ${ }^{10}$ producidos en los contextos se expresan con claridad en el siguiente relato de las consecuencias de la erupción volcánica en Chile:

“...La pérdida total de su hábitat y de sus respectivos hogares (...) conllevó a la desaparición del 80\% del lugar en donde ellos vivían. Siendo brutalmente desarraigados de sus orígenes, de sus espacios cotidianos, de sus tradiciones y de sus rutinas significativas (...) causadas por esta erupción volcánica..." (Erupción volcánica $X$ región de Los Lagos en Chile, 2008).

Las experiencias propias, cuando las ocupaciones se ven interrumpidas o modificadas en su forma ocupacional, son traídas frecuentemente al presente $y$ adquieren profundo significado en la reflexión. Una de las participantes, quien vivió el terremoto de México de 1985, lo revive con los ojos de la niña que era entonces y expresa cómo un juego, que era habitual con su mamá, cambió sustancialmente su forma y tomó la función de ocupación de protección:

“...Un día por la mañana mi papá, como todos los días, entró a bañarse mientras mi mamá y yo estábamos en mi cama jugando. De repente, siento que mi mamá me aprieta entre sus brazos y observo que el foco se mueve de un lado a otro y cada vez es más fuerte..."

(Terremoto de México, 1985).

El surgimiento de nuevas ocupaciones que congregan a grupos no afectados

\footnotetext{
${ }^{9}$ Gran Nevada de Santa Cruz, también llamada "la nevada del siglo", se trata de la nevada de mayor magnitud ocurrida en 1995 en Santa Cruz, la provincia más austral de la República Argentina.

${ }^{10}$ Cambio o giro radical en cualquier ámbito.
} 
directamente por el evento, mueve a la acción como expresión de solidaridad y en respuesta a la natural necesidad de brindar ayuda; esto amerita su análisis desde las perspectivas sociopolítica y ocupacional.

La participación activa y la organización de la comunidad, como fenómeno emergente impulsado por la necesidad común de información, de verdad y de justicia, ha sido analizada por los participantes desde su alcance ocupacional. La frecuencia de aparición ante circunstancias de riesgo y desastres, y las características comunes, permiten afirmar que favorecen el desarrollo de la movilización social y el empoderamiento a través de la ocupación de formar parte de grupos y organizaciones sociales que tienen un objetivo claro de defensa de sus derechos. Así lo expresan los siguientes relatos:

En Cusco, Perú: “...Desde hace tiempo los pobladores cusqueños reclaman ante el uso inadecuado de los recursos ambientales en varias zonas del departamento, relacionado con la actividad minera, que afecta terrenos comunales, caminos y bosques. Existen registros de demandas y reclamos que intentaron frenar proyectos de desarrollo impulsados por el gobierno junto con empresas privadas, ya que los mismos no respetan los derechos humanos de desarrollo comunitario y medio ambiente, y obligan a los pobladores a desplazarse involuntariamente..." (Terremoto en Cusco, Perú, 2007).

En Rosario, Santa Fe, Argentina: "... las rutas y accesos a Rosario estaban cortados por el agua y por los vecinos que salieron a reclamar por sus pérdidas materiales y sentimentales, muchos evacuados, las personas se quedaron sin un techo donde vivir y con ausencia de respuesta..." (Inundaciones de Melincué, provincia de Santa Fe, Argentina, década de 1970).

Los relatos también dan cuenta del surgimiento de grupos de voluntarios transitorios:

En Armero y localidades del departamento del Tolima, Colombia (1985), durante la emergencia generada por la erupción volcánica: "...Muchos de ellos víctimas y sobrevivientes de la misma tragedia, los cuales cumplieron un papel esencial en el rescate rápido y oportuno de muchos sobrevivientes, puesto que este desastre ocurrió siendo las 11:30 de la noche, dificultándose de manera significativa la atención rápida y oportuna a las autoridades competentes, debido, según ellos, a las limitantes topográficas..." (Erupción volcánica en Tolima, Colombia, 1985).

En Chaitén, $X$ región de Los Lagos en Chile, afectada también por la erupción de un volcán que lleva su nombre: "...También es importante señalar que las personas que se encuentran trabajando en la evacuación de los pobladores, son parte de la población de Chaitén y no pueden acompañar a sus familiares, empujados a ejercer otros roles..." (Erupción volcánica en $\mathrm{X}$ región de Los Lagos en Chile, 2008). 
Tras el terremoto de Pisco, Perú: "...Un grupo organizado de la misma comunidad fue a apoyar y favorecer la reconstrucción y rehabilitación de aquellos que dentro de su comunidad eran los más desprotegidos o vulnerables..." (Terremoto en Cusco, Perú, 2007).

En Santa Cruz, Argentina, tras la gran nevada: "...Podríamos ubicar esta nevada en el nivel I en las localidades más urbanizadas y nivel II en las localidades rurales, generando cambios internos en la estructura, roles y tareas de las instituciones, la convocatoria a voluntarios y ONG de participación comunitaria ciudadana..." (Nevada en Santa Cruz, provincia de Argentina, 1995).

Emerge la solidaridad como un aspecto ligado a la necesidad común y a la capacidad empática predominante durante la emergencia. En Santa Fe, Argentina, tras la inundación producida por el desborde del río Salado, reflexionaba una alumna:

"...La solidaridad durante este suceso fue enorme, solidaridad de nuestro país, de nuestras provincias y de los mismos habitantes de la ciudad de Santa Fe..." (Inundación en Santa Fe, provincia de Argentina, 2003).

En tanto, en la Ciudad de Castelli, Chaco, Argentina, frente a las sequías: "...Desde el centro de estudiantes del colegio organizamos donaciones de bidones de agua y agua lavandina, y enviamos un camión a esta provincia...".

\section{El contexto y su impacto en la ocupación}

Los cambios en las ocupaciones, rutinas y hábitos emergieron como consecuencia de la modificación del contexto. A partir del relato de uno de los participantes, acerca de la situación de millones de personas en Colombia, se introduce la concepción de desplazamiento forzado dentro de la categoría de desastres, abriendo el espectro por sobre la concepción más arraigada y tradicional de desastres de origen natural.

“...Este fenómeno social en Colombia es preocupante y alarmante (...). Dicho fenómeno social ocasiona una ruptura abrupta en estas poblaciones de sus raíces familiares, socioculturales (costumbres, hábitos, intereses, imaginarios sociales, etc.), económicas y políticas..." (Desplazamiento forzado, Colombia).

El contexto es visibilizado como el escenario primordial donde transcurre la vida ocupacional de las personas, pudiéndose reconocer la forma en que la presencia/ausencia de elementos y las modificaciones, tanto en su dimensión física como humana, impactan a las personas. Se reconoce que estos cambios jamás son por elección propia, lo cual otorga mayor dramatismo a la situación. Las modificaciones que producen los desastres en los contextos de desempeño ocupacional configuran nuevos escenarios, que la mayoría de las veces restringen oportunidades de participación ocupacional ocasionadas por daños y pérdidas materiales y simbólicas, tal como lo ejemplifica el siguiente relato: 
“...Es por estas circunstancias que estas poblaciones desplazadas llegan inmigrando a las intermedias y grandes ciudades, sin nada para comenzar, sin contar en la mayoría de veces con un trabajo, sin sus pertenencias, sin contar con una casa para alquilar o sin tener (...) parientes que les puedan ayudar inicialmente, mientras se adaptan al nuevo contexto social. Ellos llegan arriesgándose y metafóricamente casi desnudos, solamente con la vestimenta que tienen puesta. Muchos llegan después de haber caminado cientos de kilómetros, otros llegan a la terminal de transportes sin saber a dónde ir o qué hacer, y otros llegan por la solidaridad de algunos choferes o camioneros para acercarlos a cualquier otro lugar que sea más seguro para sus vidas, teniendo en estos casos que empezar su proyecto de vida desde cero, para lo cual, Ilegan en muchos casos a ocupar los semáforos y las calles en la necesidad del rebusque, la mendicidad, la prostitución, o en casos extremos, la delincuencia, para poder sobrevivir; teniendo en muchas ocasiones que vivir al aire libre, debajo de los puentes vehiculares o peatonales, parques y plazas, y los que corren con mayor suerte, ocupan y construyen techos con cartón o material reciclado en suburbios o invasiones ubicadas en el perímetro urbano de la ciudad, sin contar con ninguna infraestructura básica de saneamiento..." (Desplazamiento forzado, Colombia).

Las percepciones en tanto conjugan conciencia, experiencia, factores y ac- tuaciones personales, no siempre son coincidentes ni necesitan serlo. Se ha podido captar en este análisis que los cambios en los contextos son percibidos de manera diferente según las historias y experiencias de los habitantes del lugar. Tal es el caso de lo sucedido en Santa Cruz con la Gran Nevada. En ese lugar se da la particularidad de que confluyen dos poblaciones de origen diferente: la que originalmente habitó la zona, en su mayoría inmigrantes europeos corridos por los horrores de la guerra, y la de más reciente radicación, que emigraron de otras provincias del país. Una participante expresa:

"...Distinto es el impacto en las personas oriundas del lugar, la nevada los conecta con recuerdos y vivencias de alegría, juegos con la nieve, armado de trineos caseros..." (Nevada de Santa Cruz, Argentina, 1995).

La condición de originarios del lugar y las experiencias vividas actúan como factores amortiguadores de la percepción de pérdida de control, porque los conecta con ocupaciones altamente placenteras y significativas, organizadas en torno a la nieve, mientras que para el otro grupo de pobladores, la percepción central es de incertidumbre y crisis, llegando a poner en duda la decisión de permanecer en el lugar.

\section{Modificaciones en las rutinas ocupacionales}

Varios participantes mencionan orquestación de las ocupaciones en pos de la resolución de las necesidades más urgentes, fenómeno que configura un tipo de rutina ocupacional caracterizada por la simultaneidad en 
la realización de ocupaciones. Este fenómeno siempre conlleva a una sobrecarga ocupacional que, con el transcurrir del tiempo y si se consolida como un patrón sostenido, tendrá impacto sobre la salud de las personas.

Aparecen las ocupaciones "ad hoc" organizadas y ejecutadas en forma grupal:

"....De inmediato se armó una red solidaria que surgió naturalmente: preparar comidas, buscar ropas, alojar gente. Los jóvenes ayudaban a evacuar gente y sacar muebles (los que podían hacerlo)...".

Se puede observar y reconocer en el relato anterior y en los siguientes, la desorganización en las rutinas de las personas, incorporando nuevas ocupaciones en respuesta a la emergencia. Estas pueden resultar muchas veces disfuncionales en tanto permanezcan en el tiempo con esas características, debido a la improvisación y la ausencia de preparación:

"...Mucha gente tratando de hacer rápido lo que no hace habitualmente y en un medio decididamente no familiar...".

"...Fernanda tuvo en este caso que cambiar su rutina diaria, vivir alrededor de cuatro meses en otro barrio y dejar su hogar, afrontando la pérdida de todos sus objetos personales y los efectos emocionales que resultan de estas pérdidas...".

“...Cómo fue llegar a mi lugar de trabajo y ver que estaba todo perdido, pensar cómo empezar de nuevo y también en todos los alumnos que asistían diariamente a esta institución, cómo cambiaron sus rutinas al no contar con la escuela que muchas veces sirve de contención para los alumnos y también es el lugar donde los alumnos van diariamente a alimentarse, ya que en esa escuela funciona un comedor...".

\section{Aportes de Terapia Ocupacional}

Las propuestas de intervención que los alumnos hacen, dan cuenta de un claro reconocimiento de los objetos de estudio de la Terapia Ocupacional, identificando la ocupación como centro de la intervención, así como las rutinas, la promoción de los roles de participación y la estructuración de los ambientes/contextos, con especial cuidado en la atención de las condiciones particulares de salud.

Visualizan como un aspecto clave de la intervención, la participación activa de las personas afectadas, tomando como centro las necesidades sentidas e identificadas por ellas mismas y los aspectos que comunican como objetivos sobre los cuales desean trabajar.

Dentro de las intervenciones distinguen la función del terapeuta ocupacional en la promoción de la ocupación significativa; la orientación en la ubicación física de las personas con movilidad reducida; el asesoramiento para evitar enfermedades infectocontagiosas; el asesoramiento a voluntarios para evitar la recarga ocupacional y a las personas afectadas para promover rutinas que apoyen el progreso de la adaptación; y la generación de espacios de diálogo que posibiliten, a través de la comunicación, expresar 
emociones y necesidades, así como poner en común las habilidades de cada uno para poder colaborar en las tareas propias del mantenimiento del lugar donde se reside. Hay una valoración de la importancia del trabajo en equipo, con un reconocimiento de los aspectos distintivos sobre los cuales el terapeuta ocupacional puede enriquecerlo: rutinas, ocupaciones significativas, lugar de control de las personas sobre la situación, recarga ocupacional.

Se evidencia la incorporación de nuevas herramientas teóricas que permiten ampliar el campo de acción de Terapia Ocupacional, cruzando las bases de esta última con otros constructos de reconocimiento y aplicación internacional. Como ejemplo, las palabras de una alumna respecto de la utilidad que podría tener contar en la profesión con algún protocolo de intervención de Terapia Ocupacional para situaciones de emergencias complejas y desastres:

“.....pensaba, que estaría bueno, que nunca lo vi, una guía de las posibles actividades que un TO puede llevar a cabo en estas situaciones, o ejemplos de diferentes actividades, por más que los escenarios sean diferentes...".

Todo lo anterior tiene relación directa con lo que plantea Ronsenfeld en el Modelo para intervenciones en crisis, cuyo objetivo es mejorar el comportamiento de afrontamiento de crisis. Algunas de las evaluaciones que plantea la autora para emprender una intervención exitosa, son las siguientes: cuestionarios de la vida diaria y evaluaciones funcionales, la historia ocupacional, los intereses vocacionales, la historia educativa y social, las cuales proporcionan una importante base de datos contextuales a partir de los cuales se establece el concepto de presente del individuo, conociendo su estado emocional y el ambiente, entre otros elementos (Rosenfeld, 1982).

Los terapeutas ocupacionales, a través de la ocupación, contribuimos a los esfuerzos de las comunidades afectadas en la rehabilitación y reconstrucción luego de un desastre. En este sentido, el equipo de docentes investigadores que llevó adelante este curso de posgrado, a partir del recorrido realizado desde el 2003 hasta la actualidad, desarrollando diferentes investigaciones en la temática, sostiene que la intervención del terapeuta ocupacional debe efectuarse durante todo el ciclo de desastres, ya que la ocupación está presente en la vida de los seres humanos y resulta un soporte efectivo. A partir de los proyectos de investigación se ha demostrado el potencial sinérgico de la ocupación humana en sus múltiples expresiones y las contribuciones que podemos hacer como profesionales en problemáticas de desastres y emergencia (Boffelli et al, 2015).

\section{Recapitulación y aprendizaje}

En el primer módulo, el requerimiento de recuperar eventos relacionados con la propia historia funciona como factor motivador del posterior análisis a la luz de la bibliografía proporcionada; la tendencia a entremezclar lo anecdótico y vivencial con la teoría, lejos de quitarle profundidad a las interpretaciones, las carga de humanidad y comienza a perfilar un estilo propio 
de cada participante para construir conocimientos. Estas particularidades en los estilos individuales (frecuencia y calidad de las participaciones, modos de abordar los temas, cuestionamiento de los propios preconceptos, etc.) se volverían reconocibles para los docentes conforme el avance del curso.

\section{La participación en el curso como ocupación}

Merece una mención especial dentro de la categoría Ocupación, el desempeño de los propios alumnos del curso en la actividad presente: participar en un curso virtual de preparación y respuesta a desastres.

Aunque para muchos participantes esta no era la primera experiencia en formación virtual, el entorno, en ocasiones, plantea algunas pequeñas dificultades que se van salvando en el transcurso mismo de su desarrollo:

"...Estuve mirando la actividad evaluativa del módulo... pregunto cómo me conecto con M.B y G. para intercambiar y acordar las respuestas. Tenemos algún espacio en la plataforma...?"

"... ¿El correo interno de plataforma es a través del foro de intercambio del módulo, cierto? Otro no encuentro... igual les paso mi correo por si además nos comunicamos por ahí..."

La actividad de preparar la evaluación del módulo en forma grupal presenta todo un desafío, que es interpretado como muy positivo y enriquecedor.

En general todos coinciden en que la participación en el curso les insume un tiempo considerable, debido principalmente a la cantidad de bibliografía ofrecida; pero manifiestan que, tanto los temas como los recursos utilizados, resultan altamente motivadores, así como la relación personal que se va consolidando entre los diferentes participantes. Algunos de los intercambios dan cuenta de lo anterior:

“...Acá estoy tratando de ponerme al día tanto con el material como con el entorno virtual...".

“...Es como que necesito leer y releer este texto. Nos convoca en estos tiempos a la participación activa, el trabajo colectivo...".

“...Les decía que a veces me paraliza el tema, pero me sirve mucho como ustedes lo presentan... canciones, videos, entrevistas, diferentes formatos que van haciendo que tomemos aire... como la tierra....".

“...Espero que tus cosas estén mejor... Te sumamos al grupo y vamos intercambiando dudas!! Yo también aún estoy leyendo... Besos!!...".

El hecho de no encontrar un único punto de vista en los materiales ofrecidos, así como la apertura a otros recursos, los mueve a la reflexión crítica sobre sus propias concepciones:

"...Lo interesante, a mi parecer, (...) fue el observar la relatividad de las apreciaciones y el ver dos documentales, en teoría pro ambientalistas, pero con claras diferencias. Me sirvió para entender las dos posturas y así poder crearme una visión más amplia...". 
El contacto con el marco de justicia ocupacional y la perspectiva de derechos humanos, constituye para muchos un viraje conceptual que los lleva a la reflexión acerca de las propias prácticas y situaciones vividas en el ejercicio cotidiano:

"...El marco teórico de la Justicia Ocupacional no lo conocía en profundidad...es muy claro en lo ideológico, pero el punto de la atribución del poder a través de la ocupación me abrió la cabeza... sigo pensándolo...".

Los nuevos conocimientos promueven un viraje en el razonamiento profesional de los alumnos, invitando a reflexionar sobre el papel central del terapeuta ocupacional en nuevos contextos de intervención, que se configuran como escenarios emergentes de práctica profesional, en los cuales, lo central y clave es el paradigma crítico desde el cual se produzca la lectura de la situación y el posicionamiento político que se adopte:

“...Tampoco nunca había pensado a la gestión de riesgo como un derecho humano y la responsabilidad de los actores sociales en la protección de los ciudadanos. Y allí también pienso en los TO como animándonos a ocupar espacios de decisión pública, espacios de poder para transformar...".

\section{Conclusiones}

La evaluación de los participantes sobre la propuesta académica destaca la vigencia y pertinencia del tema, la calidad académica, así como la accesibilidad y flexibilidad de la propuesta.
Así mismo, el diseño metodológico y la propuesta pedagógica confluyeron para otorgar validez a la propuesta político-educativa, posible de replicarse a futuro con profesionales de otras áreas. Se destaca el alto nivel de desempeño académico de los participantes (de muy bueno a excelente), como uno de los indicadores de impacto, considerando que para todos fue el primer contacto con los cuerpos teóricos y los enfoques desarrollados en el curso (Marco de justicia ocupacional, Enfoque de derechos humanos y Gestión de riesgo de desastres).

La calidad de las intervenciones y propuestas revelan que se pone en funcionamiento una red de relaciones entre los recursos del entorno, las decisiones pedagógicas, el tema convocante, los contenidos y las teorías que retroalimentan positivamente la motivación, el interés por el conocimiento y la participación pro-activa de los alumnos.

Para los participantes, los foros y las actividades grupales resultaron las propuestas más eficaces y motivadoras. Estas constituyen verdaderas "canteras" de producción de conocimientos. Los alumnos valoraron positivamente las propuestas colaborativas y cooperativas, que modifican el esquema tradicional de transmisión de conocimientos por el proceso de construcción social. Las actividades grupales presentaron cierta dificultad por la falta de experiencias previas y el tiempo que requieren. Se destaca que son motivadoras de nuevos aprendizajes.

Todos los profesionales participantes identificaron que se abre un nuevo escenario para la intervención profe- 
sional. Los nuevos conocimientos promueven un viraje en el razonamiento, rompe con los modos tradicionales y tensiona saberes y prácticas. Se plantea como ineludible un posicionamiento político claro respecto de la ocupación como derecho, la justicia ocupacional, las diferentes formas de injusticia ocupacional y el apartheid ocupacional.

Las ocupaciones emergen con multiplicidad de formas, funciones, significados y contextos, acorde a los planteamientos de las ciencias de la ocupación (Clark et al., 1991). Los participantes lograron realizar análisis a nivel macro, meso y micro; identificar las características centrales de las ocupaciones en las diferentes instancias del ciclo de desastre; y los servicios y acciones pertinentes para cada situación. Se elaboraron propuestas de intervención profesional para diferentes momentos y contextos, con énfasis en lo local.

También se concluye que se ha aportado a la consolidación de una identidad profesional centrada en el valor y la potencialidad de cambios de las ocupaciones, y en la reivindicación de las mismas como una necesidad y un derecho humano.

El recorrido realizado por este equipo de profesionales, investigadores y docentes de Terapia Ocupacional, pone en evidencia que el mismo se ha constituido en un espacio de formación de recursos y consulta para alumnos de Terapia Ocupacional y otras carreras; ha desarrollado capacitaciones para profesionales, estudiantes y miembros de la comunidad.
El equipo ha realizado transferencia actuando como consultor de diferentes organismos, entre ellos el Centros de Estudios para Políticas Municipales y Provinciales, y de colegas de países como Perú, Brasil y Chile. Brindó información de apoyo al creador de un sistema de información para catástrofes y es uno de los impulsores de la constitución de la Red Latinoamericana T.O. en Alerta, junto con TO Alerta Chile, esta red quedó formalmente constituida en el marco del Congreso Latinoamericano de Terapia Ocupacional, realizado en San Pablo, Brasil, en el año 2011. El objetivo central de la red es construir un espacio que permita intercambiar y compartir experiencias e información y desarrollar líneas de trabajo, capacitación, investigación y preparación de terapeutas ocupacionales para la intervención en situaciones de desastres. Esta red funciona predominantemente de manera virtual, aprovechando las ventajas que brindan las tecnologías de la comunicación, y se aloja en el sitio web INTOCA: Intervención e Investigación de Terapia Ocupacional en Catástrofes, www.intoca.com.ar

En cuanto a publicación y difusión, se realizaron presentaciones a nivel local, nacional e internacional (Santa Fe, Mar del Plata, Colombia, India, Perú, Chile, México y Brasil). Adicionalmente, el equipo obtuvo premios y menciones en eventos científicos de índole local, nacional e internacional.

Así, la Federación Mundial de Terapeutas Ocupacionales (WFOT) ha reconocido a este equipo como referente latinoamericano de Terapia Ocupacional en catástrofes, por los 
aportes realizados en la investigación y la práctica, así también como la representación por Argentina en el proyecto global denominado WFOT Disaster Preparedness and Response.

\section{Referencias}

Beck, U. (2000). Retorno a la teoría de la sociedad del riesgo. Boletín de la Asociación de Geógrafos Españoles, (30), 9-20.

Beck, U. (2002). La sociedad del riesgo global. Barcelona: Siglo XXI Editores.

Blaikie, P., Cannon, T., David, I. \& Wisner, B. (1996). Vulnerabilidad: el entorno social, económico y político de los desastres. La Red. Recuperado de: http://www.desenredando.org/public/libros/1996/vesped/ vesped-biblio_sep-09-2002.pdf

Boffelli, M., Boggio, C., Chiapessoni, D., Demichelis, M., Demiryi, M.M., Heit, M.C. (2015). Listening to the voices of survivors: the floods of 2003 in Santa Fe, Argentina. En: Rushford, N. \& Kerry, T. (Ed.). Disaster and development. An occupational perspective (pp.53-62). Elsevier Health Sciences

Clark, F., Parham, D., Carlson, M., Jackson, J., Pierce, D., Wolfe, R. \& Zemke, R. (1991). Occupational Science: Academic innovation in the service of occupational therapy`s future. American Journal of Occupational Therapy, (45), 300-310.
Fetterman, D.M. (2001). Foundations of Empowerment Evaluation. Londres: Sage

Gascón, M. (2005). Vientos, terremotos, tsunamis y otras catástrofes naturales. Historia y casos latinoamericanos. Buenos Aires: Biblios,

Giddens, A. (1993). Consecuencias de la modernidad. Madrid: Alianza.

Huertas, J. (1976). La clasificación de los objetivos de aprendizaje. Su función y utilidad. México: Trillas.

Lavell, A. (2005). Elementos conceptuales para la prevención y reducción de daños originados por amenazas socionaturales. Cuatro experiencias en América Latina y el Caribe. Cuadernos de la CEPAL (91). Recuperado de: http://www.eclac.org/ publicaciones/xml/1/23711/lcg2272e. pdf.

Rosenfeld, M. S. (1982). Modelo de intervención por medio de la actividad en comunidades afectadas por desastres. American Journal of Occupational Therapy, 36 (4), 1-11

Valdés, J. (2006). La reducción del riesgo de desastres: un llamado a la acción. EIRD Informa - América Latina y el Caribe, (13). Recuperado de: http://www.eird.org/esp/ revista/no_13_2006/art5.htm 